\title{
Angiosarcoma Involving Solid Organs and the Gastrointestinal Tract with Life-Threatening Bleeding
}

\author{
Chiaki Maeyashiki $^{a} \quad$ Naoyoshi Nagata $^{a}$ Naomi Uemura $^{b}$ \\ ${ }^{a}$ Department of Gastroenterology and Hepatology, National Center for \\ Global Health and Medicine, Tokyo, and ${ }^{\mathrm{b}}$ Department of Gastroenterology \\ and Hepatology, National Center for Global Health and Medicine, Kohnodai \\ Hospital, Chiba, Japan
}

\section{Key Words}

Angiosarcoma - Hemangiosarcoma - Gastrointestinal tract · Endoscopic biopsy · Capsule endoscopy - Endoscopic ultrasound-guided fine needle aspiration biopsy . Double balloon endoscopy · Laparoscopic surgery

\begin{abstract}
We report a rare case of angiosarcoma involving the gastrointestinal tract, liver, spleen, pancreas, kidney, lung and vertebrae, as well as bulky dissemination in the pleuroperitoneal membranes. A 72-year-old man with no history of illness became aware of melena. Laboratory findings revealed anemia, and upper gastrointestinal endoscopy revealed multiple reddish nodules in the stomach and duodenum. However, biopsy specimens showed no evidence of histological features. Computed tomography and fluorodeoxyglucose positron emission tomography showed space-occupying lesions in the spleen, liver and vertebrae. Angiosarcoma was diagnosed by endoscopic ultrasound and fine needle aspiration biopsy of the spleen and repeated endoscopic biopsy of the stomach. We performed laparoscopic splenectomy to avoid rupture of the involved spleen. Due to continuous gastrointestinal bleeding, double balloon endoscopy was performed and multiple bleeding lesions were detected throughout the small intestine. Surgical hemostasis was performed by partial enterectomy, but anemia continued to worsen. Therefore, we conducted transcatheter arterial embolization. Despite attempting several modalities and frequent daily blood transfusion, the anemia did not improve, and the patient expired due to hemorrhagic shock. Subsequent autopsy revealed the cause of death as rupture and bleeding due to disseminated involvement of the small intestine.
\end{abstract}




\section{Introduction}

Angiosarcoma is a rare malignant neoplasm representing less than $2 \%$ of all soft tissue sarcomas [1]. The spleen and liver are the most commonly involved sites, and only a few reports of gastrointestinal tract involvement have been published [1]. Definitive diagnosis of angiosarcoma has only been possible by exploratory laparotomy and splenectomy because biopsy is contraindicated because of a high risk of rupture [2]. Moreover, no standard therapeutic method exists for angiosarcoma, and its prognosis is poor [3]. Most patients have a fulminant clinical course and a short mean survival time [3]. Here, we report a rare case of angiosarcoma who presented with life-threatening bleeding and involvement of the gastrointestinal tract (stomach, duodenum, jejunum and ileum), liver, spleen, pancreas, kidney, lung and vertebrae, as well as bulky dissemination in the pleuroperitoneal membranes.

\section{Case Report}

A 72-year-old man with a chief complaint of dizziness and black stools arrived at a hospital by ambulance. The patient was a retired architect and builder who had spent 50 years in the construction industry, and he had neither a medical history nor a family history of malignant cancer. Blood test results indicated severe anemia ( $\mathrm{Hb} 3.7 \mathrm{~g} / \mathrm{dl})$. Upper gastrointestinal endoscopy detected several elevated bright-red lesions in the stomach (ig. 1a) and the duodenum (fig. 1b), but biopsy pathology findings were normal.

Since anemia and black stools persisted, the patient was transferred to our hospital 10 days after the initial examination at the first hospital. Upper gastrointestinal endoscopy with biopsy was performed twice, but again biopsy findings were normal. Computed tomography and fluorodeoxyglucose positron emission tomography, which were performed to search for the development of lesions in other organs, revealed multiple masses in the spleen, liver and thoracolumbar spine. The splenic masses in these organs were larger than those in other organs. Endoscopic ultrasound-guided fine needle aspiration biopsy (EUS-FNAB) was performed via the stomach to obtain splenic mass specimens. Hematoxylin-eosin staining revealed clusters of tumor cells (fig. 2a; $\times 20$ magnification), and immunohistochemical staining identified them as positive for CD31 (fig. 2b; $\times 10$ magnification), CD34 (fig. 2c; $\times 10$ magnification) and factor VIII (fig. $2 \mathrm{~d} ; \times 10$ magnification). On the basis of these findings, angiosarcoma was diagnosed on hospital day 37 . A fourth upper gastrointestinal endoscopic biopsy was performed to make a definitive diagnosis, and immunostaining showed similar tumor cell clusters, leading to a diagnosis of gastric angiosarcoma. Anemia and black stools persisted, suggesting the presence of lesions in other gastrointestinal tract organs. Indeed, capsule endoscopy detected elevated bright-red lesions in the jejunum and ileum (fig. 1c), which were thought to be the cause of the anemia.

A high risk of splenic rupture was indicated, so endoscopic splenectomy was performed as a preventive measure on hospital day 58. A double balloon technique was used for endoscopic hemostasis, and multiple bleeding lesions were confirmed in the small intestine (fig. 1d). Surgical hemostasis was performed by partial enterectomy to remove the lesions with severe bleeding on hospital day 86. Since the effects of this surgical treatment on anemia and on the production of black stools were negligible, transcatheter arterial embolization was performed via the superior mesenteric artery. Despite all efforts, gastrointestinal bleeding and the accompanying anemia persisted. The patient could not undergo chemotherapy and needed daily blood transfusions during his hospital stay, but despite receiving 92 units of red cell concentrate during his hospital stay, he eventually expired due to hemorrhagic shock on hospital day 103.

The subsequent autopsy revealed angiosarcoma in the stomach, duodenum, jejunum, ileum, liver (fig. $3 \mathrm{a}$ ), spleen (fig. 3b), pancreas, kidney, lung and vertebrae, as well as bulky dissemination in the pleuroperitoneal membranes (fig. 3c). The cause of death was determined to be rupture and bleeding due to disseminated involvement of the small intestine. 


\section{Discussion}

Angiosarcoma is a rare disease: only $2 \%$ of all sarcomas constitute the angiosarcoma subtype [1]. Exposure to thorium dioxide, vinyl chloride and arsenic has been implicated in the cause of this disease [1]. Considering the long-term involvement of our patient in the construction industry, he might have been exposed to these chemicals for a considerable time.

The first recorded angiosarcoma, found in the spleen, was reported by Langhans et al. in 1879 [2]. Since then, only a limited number of cases of gastrointestinal angiosarcoma have been reported [2], and the present case is the first to show angiosarcoma lesions in the stomach, duodenum, jejunum, ileum, liver, spleen, pancreas, kidney, lung and vertebral body.

Although upper left abdominal pain has been reported in $80 \%$ of splenic angiosarcoma cases, typical symptoms of gastrointestinal angiosarcoma remain unclear [1]. The present patient had painless splenic lesions, and it was the production of black stools that prompted a series of tests, which led to angiosarcoma being diagnosed.

Diagnosis of angiosarcoma in such organs is considered to be possible only via exploratory laparotomy and splenectomy [2]. However, in the present case, diagnosis was made by EUS-FNAB and frequent endoscopic biopsies, indicating the utility of these techniques in the diagnosis of angiosarcoma. No serious complications of these biopsy procedures, such as bleeding or perforation, were observed in our case.

EUS-FNAB has become widely established as an effective means for diagnosing tumors, suggesting that it can replace open biopsy as the standard diagnostic modality [4]. In the present case, we performed several procedures such as splenectomy, enterectomy and transcatheter arterial embolization in an attempt to control bleeding attributed to angiosarcoma, but none were successful. The patient consequently died only 2 months after diagnosis, probably due to sudden severe bleeding.

The prognosis of angiosarcoma is poor; the median survival of splenic angiosarcoma is 5 months [5]. When angiosarcoma is suspected in multiple organs, it is desirable to monitor the patient closely for bleeding and to provide hemostatic therapy at an early stage.

\section{Acknowledgement}

We wish to express our gratitude to Hisae Kawashiro (clinical research coordinator) for assisting with data collection. This report was supported by a grant from the National Center for Global Medicine (Japan).

\section{Disclosure Statement}

The authors declare no potential conflicts of interest. 


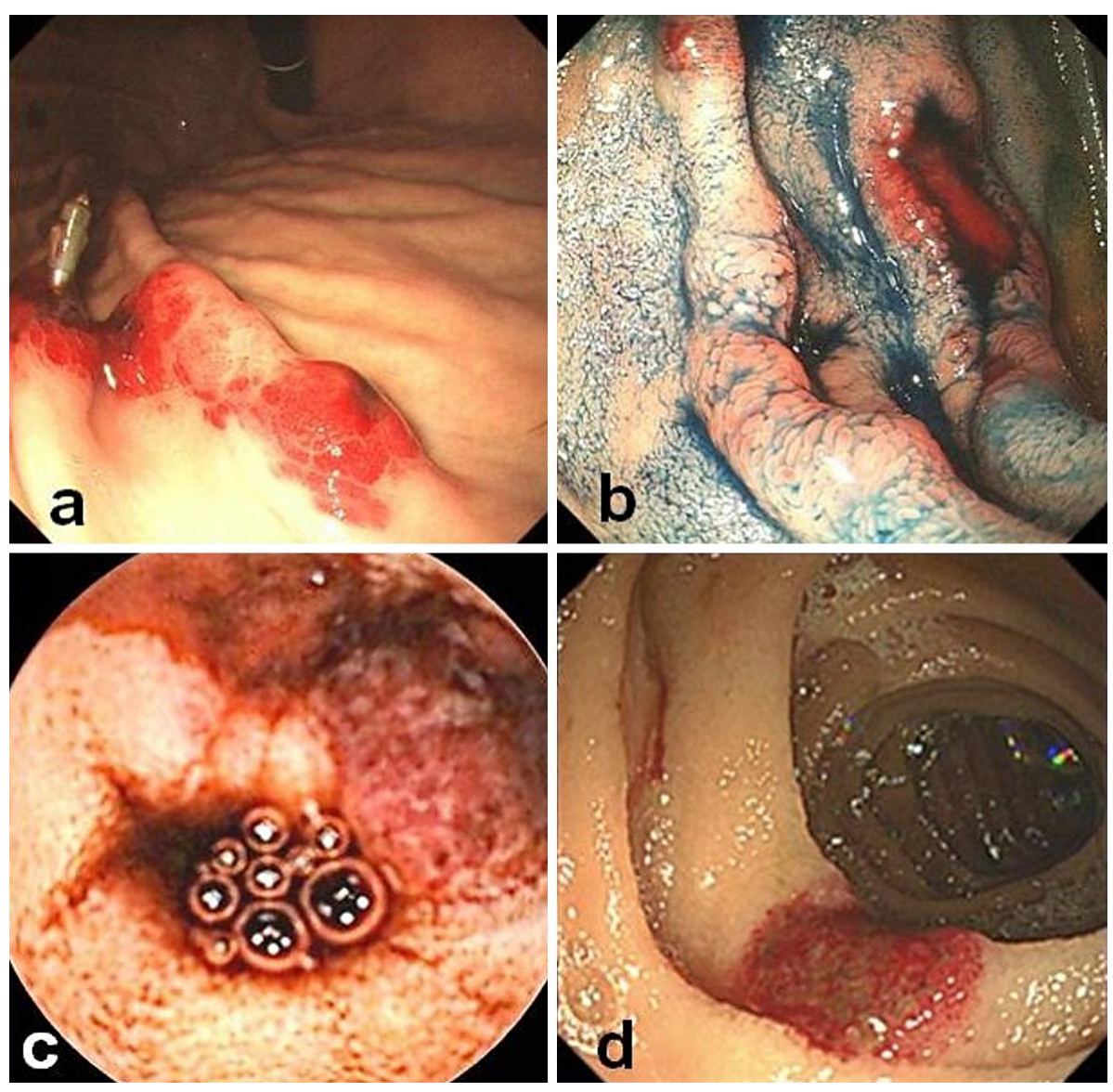

Fig. 1. Endoscopy showing gastrointestinal angiosarcoma. Upper gastrointestinal endoscopy detected reddish submucosal lesions in the stomach (a) and in the duodenum with indigo carmine dye (b). Capsule endoscopy detected elevated bright-red lesions in the jejunum and ileum (c). Double balloon endoscopy detected multiple bleeding lesions in the small intestine (d). 


\begin{tabular}{r|l|l|l}
$\begin{array}{r}\text { Case Reports in } \\
\text { Gastroenterology }\end{array}$ & $\begin{array}{l}\text { Case Rep Gastroenterol 2012;6:772-777 } \\
\text { DOl: 10.1159/000346398 }\end{array}$ & $\begin{array}{l}\text { Published online: } \\
\text { December 20, 2012 }\end{array}$ & $\begin{array}{l}\text { @ 2012 S. Karger AG, Basel } \\
\text { ISSN 1662-0631 } \\
\text { www.karger.com/crg }\end{array}$ \\
\hline
\end{tabular}
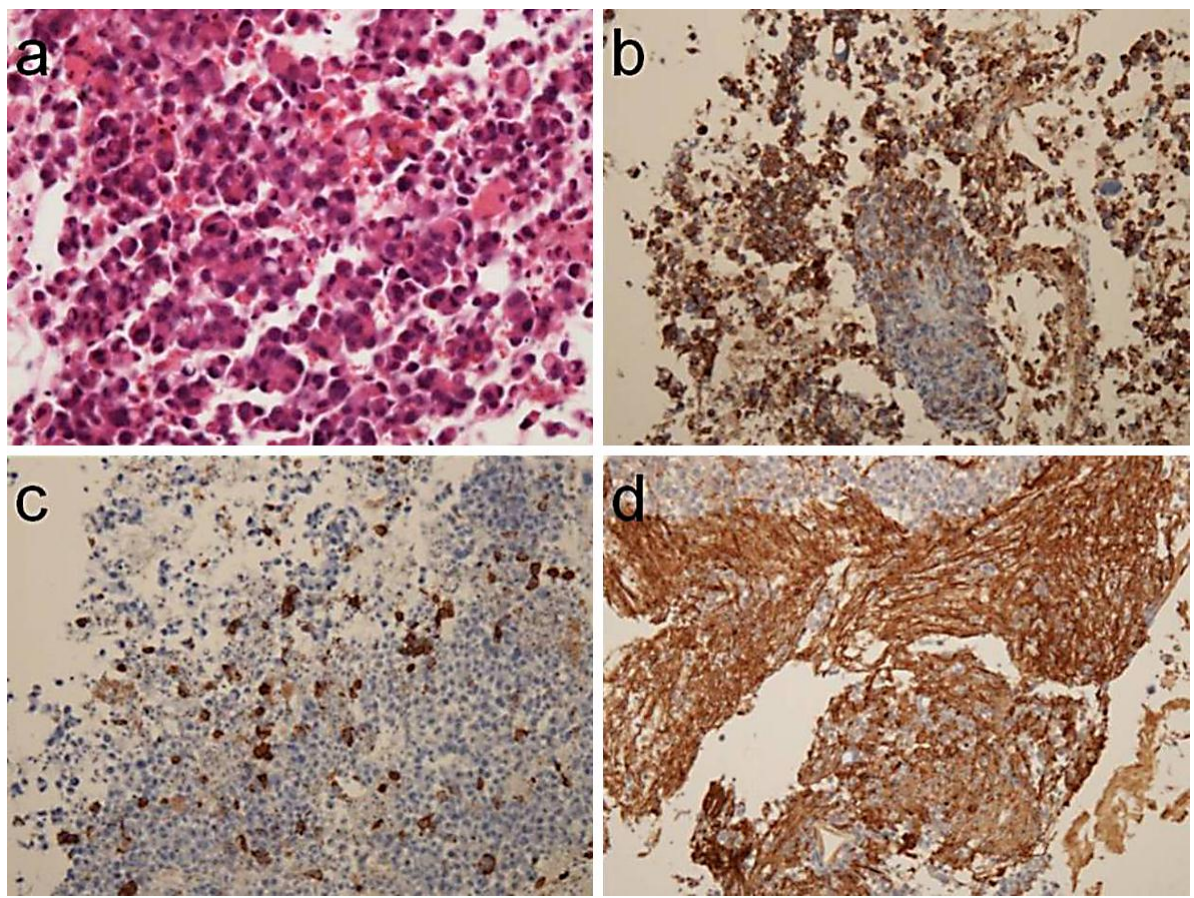

Fig. 2. Pathological features of splenic angiosarcoma. Hematoxylin-eosin staining revealed clusters of tumor cells (a; $\times 20$ magnification), and immunohistochemical staining identified them as positive for CD31 (b; $\times 10$ magnification), CD34 (c; ×10 magnification) and factor VIII (d; $\times 10$ magnification).
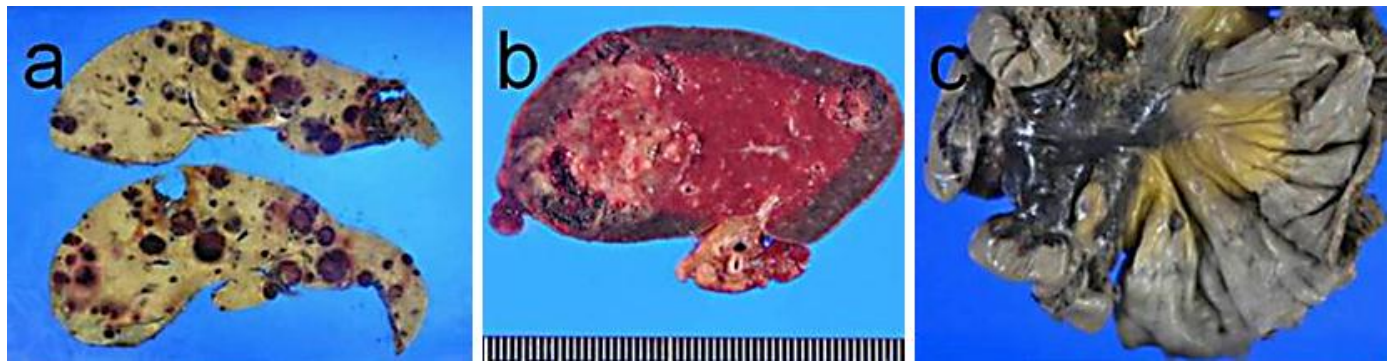

Fig. 3. Pathologic autopsy showing angiosarcoma. Multiple angiosarcomas were detected in the liver (a), a bulky tumor mass was detected in the spleen (b), and dissemination was observed in the pleuroperitoneal membranes (c). 


\section{References}

1 Ozturk E, Mutlu H, Sonmez G, Sildiroglu HO: Primary angiosarcoma of the spleen. Turk J Gastroenterol 2007;18:272-275.

-2 Delacruz V, Jorda M, Gomez-Fernandez C, Benedetto P, Ganjei P: Fine-needle aspiration diagnosis of angiosarcoma of the spleen: a case report and review of the literature. Arch Pathol Lab Med 2005;129:1054-1056.

-3 Ren T, Chen XQ: Giant diaphragmatic angiosarcoma of adult: a case report and review of literature. Case Report Med 2012;2012:950856.

-4 Polkowski M, Larghi A, Weynand B, Boustiere C, Giovannini M, Pujol B, Dumonceau JM; European Society of Gastrointestinal Endoscopy (ESGE): Learning, te chniques, and complications of endoscopic ultrasound (EUS)-guided sampling in gastroenterology: European Society of Gastrointestinal Endoscopy (ESGE) Technical Guideline. Endoscopy 2012;44:190-206.

5 Hai SA, Genato R, Gressel I, Khan P: Primary splenic angiosarcoma: case report and literature review. J Natl Med Assoc 2000;92:143-146. 